\title{
A Comparative Study on the Alterations of
}

\section{Endocytic Pathways in Multiple Lysosomal Storage}

\section{Disorders}

\author{
Jeff Rappaport ${ }^{1 \dagger}$, Rachel L. Manthe ${ }^{1 \dagger}$, Melani Solomon ${ }^{2}$, Carmen Garnacho ${ }^{3}$, and \\ Silvia Muro ${ }^{1,2, *}$
}

${ }^{1}$ Fischell Department of Bioengineering, University of Maryland, College Park, MD 207424450, USA

${ }^{2}$ Institute for Bioscience and Biotechnology Research, University of Maryland, 5115 Plant Sciences Building, College Park, MD 20742-4450, USA

${ }^{3}$ Department of Normal and Pathological Histology and Cytology, University of Seville School of Medicine, Seville 41009, Spain

${ }^{\dagger}$ These authors contributed equally to this article.

*Address correspondence to: Silvia Muro, 5115 Plant Sciences Building, College Park, MD 20742-4450. Tel: 1+301-405-4777; Fax: 1+301-314-9075; E-mail: muro@umd.edu 


\section{Supporting Information (SI)}

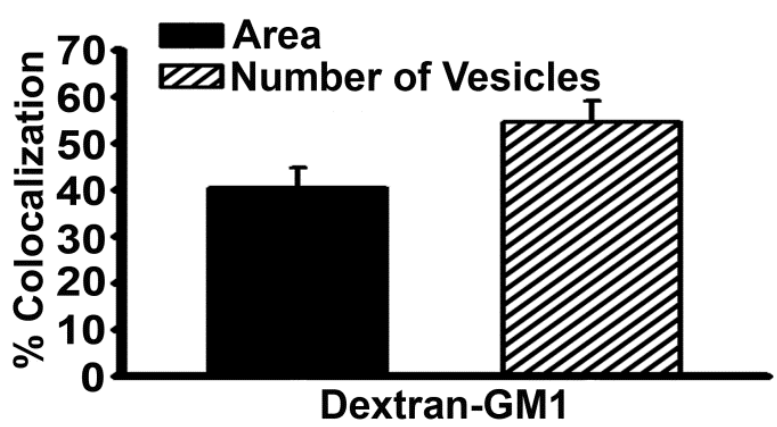

SI 1. Caveolae-mediated uptake of fluid-phase dextran. Quantification of the colocalization of Texas Red dextran-positive vesicles internalized by wild-type fibroblasts $\left(30 \mathrm{~min}, 37^{\circ} \mathrm{C}\right)$ with green fluorescent-immunostained ganglioside GM1. The graph shows percent colocalization of the area and number of vesicles occupied by these markers, analyzed by fluorescence microscopy. Data are the mean \pm SEM.

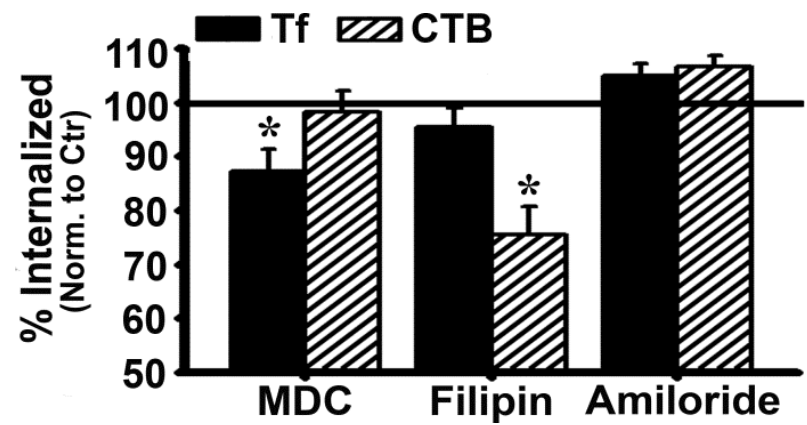

SI 2. Specific inhibition of receptor-mediated uptake of ligands. Uptake of red fluorescent transferrin $\left(\mathrm{Tf} ; 30 \mathrm{~min}, 37^{\circ} \mathrm{C}\right)$ or cholera toxin $\mathrm{B}\left(\mathrm{CTB} ; 1 \mathrm{~h}, 37^{\circ} \mathrm{C}\right)$ by wild-type fibroblasts was studied in control condition vs. the presence of monodansylcadaverine (MDC; an inhibitor of clathrin-mediated endocytosis), filipin (an inhibitor of caveolar endocytosis), or amiloride (an 
inhibitor of macropinocytosis). Cell-surface Tf or CTB were green immunostained using specific antibodies, followed by fluorescence microscopy analysis (see Figures 4 and 5). Internalization was quantified as the percentage of internalized $\mathrm{Tf}$ or CTB compared to the total (internal+surface-bound) counterparts. Data were then normalized to control cells incubated in the absence of inhibitors, indicated by the horizontal line. Data are the mean \pm SEM. ${ }^{*}$ Comparison with control cells ( $\mathrm{p}<0.05$ by Student's t-test).

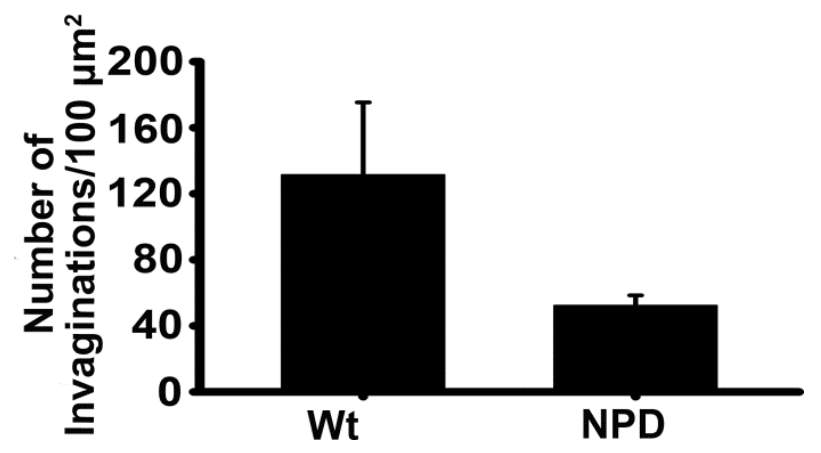

SI 3. Presence of caveoli on the surface of cell membranes. Wild-type (Wt) and NPD fibroblasts were imaged by scanning electron microscopy (SEM), whereby the number of membrane invaginations with a size $\leq 50 \mathrm{~nm}$ in diameter and void of an electron-light halo surrounding the opening of the invagination were quantified and expressed per cell-surface area. Data are the mean $\pm \mathrm{SEM}$. 


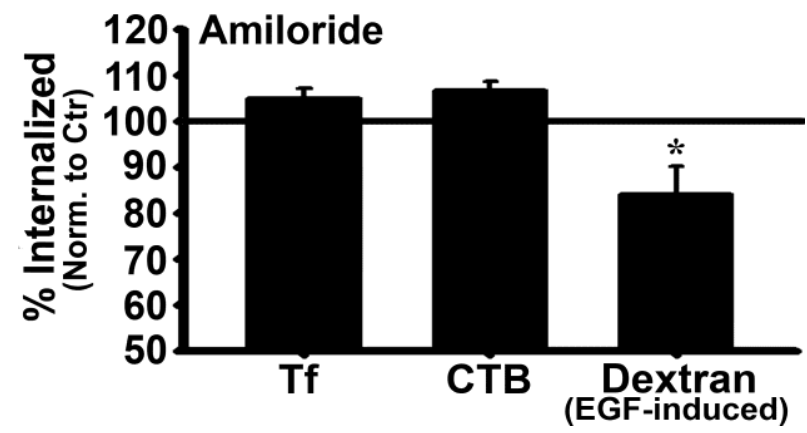

SI 4. Specific inhibition of macropinocytosis. Wild-type (Wt) fibroblasts were incubated at $37^{\circ} \mathrm{C}$ for 30 min with transferrin (Tf; clathrin route), $1 \mathrm{~h}$ with cholera toxin B (CTB; caveolar route), or co-incubated for 30 min with Texas Red dextran and EGF (macropinocytosis). Incubations were performed in the absence (control) vs. presence of amiloride, an inhibitor of macropinocytosis. Uptake of Tf, CTB, and dextran were assessed and quantified as described in Figures 4, 5, and 3, respectively. Data are normalized to the control condition, which is indicated by the horizontal line. Data are the mean \pm SEM. *Comparison with control cells $(\mathrm{p}<0.05$ by Student's t-test). 

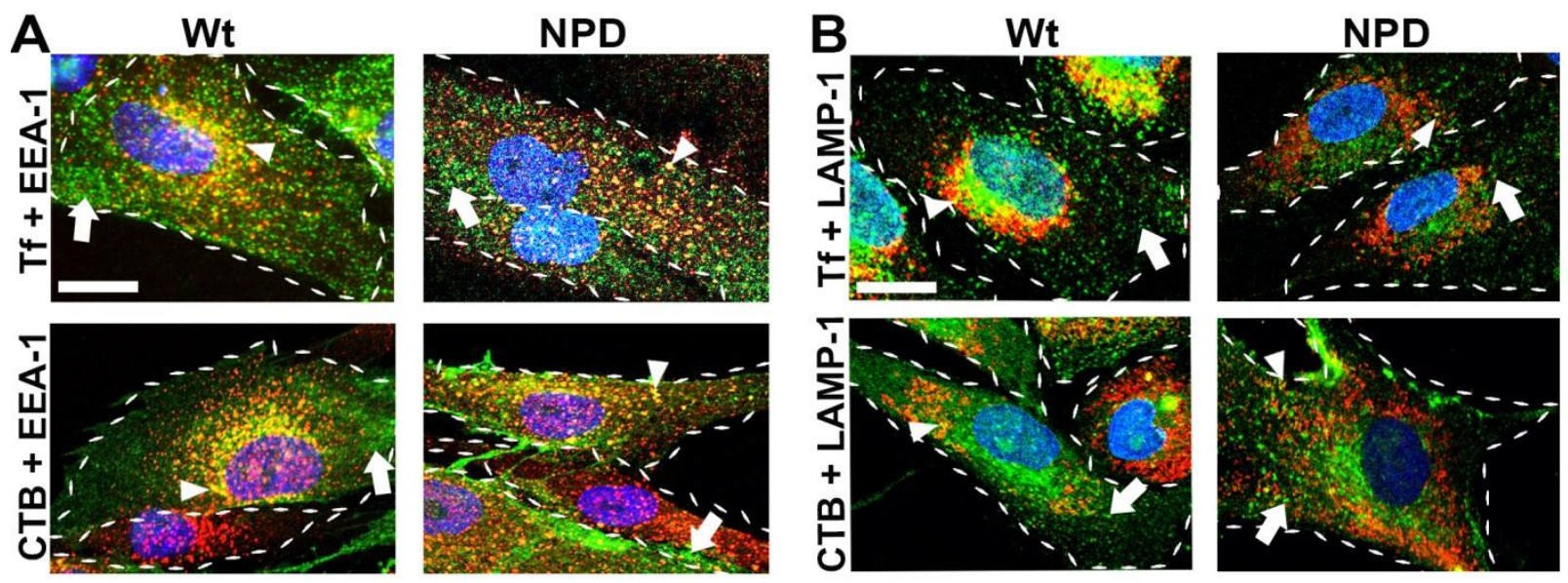

SI 5. Visualization of intracellular trafficking of ligands. Wild-type (Wt) or NPD fibroblasts were incubated for $30 \mathrm{~min}$ at $37^{\circ} \mathrm{C}$ with fluorescent transferrin (Tf) or cholera toxin $\mathrm{B}(\mathrm{CTB})$, pseudocolored green, followed by immunostaining of (A) early endosomes or (B) lysosomes using antibodies against EEA-1 or LAMP-1, respectively (pseudocolored red). Cell nuclei were stained with DAPI. Colocalization of Tf or CTB with EEA-1 or LAMP-1 appears yellow (red + green; arrowheads), while Tf or CTB localized in other areas are visualized as green (arrows). Dashed lines $=$ cell borders, as observed from phase-contrast microscopy. Scale bar $=10 \mu \mathrm{m}$. 